\title{
Novel Approach for Ballistic Targets Classification from HRRP frame
}

\author{
Adriano Rosario Persico*, Christos V. Ilioudis* Carmine Clemente* and John Soraghan* \\ *University of Strathclyde, CESIP, EEE, 204, George Street, G1 1XW, Glasgow, UK \\ E-mail: adriano.persico, c.ilioudis, carmine.clemente, j.soraghan -@ strath.ac.uk
}

\begin{abstract}
Nowadays the challenge of the identification of Ballistic Missile (BM) warheads in a cloud of decoys and debris is essential for the defence system in order to optimize the use of ammunition resources avoiding to run out of all the available interceptors in vain. In this paper a novel approach for the classification of ballistic threats from the High Resolution Range Profile (HRRP) frame is presented. The algorithm is based on the computation of the inverse Radon Transform (IRT) of the HRRP frame as target signature, and on the evaluation of pseudoZernike moments, as final feature vector. Firstly, the algorithm is presented emphasizing the characteristics of the HRRP frame due to target micro-motions. Then, the classification results on simulated data are shown for various operational conditions.
\end{abstract}

\section{INTRODUCTION}

Since the early stages of the development of Ballistic Missiles (BMs), many countries invest annually a huge budget into research and production of countermeasures in order to minimize the effectiveness of Ballistic Missile Defence (BMD) systems [1]. One of the most common practices is the use of a large number of decoys, or false targets, with the aim to confuse the defence systems. The longer part of a BM flight takes place in the exo-atmosphere and it is commonly known as the mid-course phase. The lightweight decoys are released during this phase, so both the decoys and the much heavier warhead travel on similar trajectories due to the absence of atmospheric drag in the vacuum of space [2]. In absence of reliable target identification, the defence system has to intercept all the detected targets, including decoys, in order to prevent the warhead from reaching its aim. Since the anti-ballistic missile systems have a limited number of interceptors, the challenge of Ballistic Targets (BTs) classification identifying the warhead into a cloud of decoys and debris is of fundamental importance to increase the safety level.

The capability to distinguish between warheads and decoys during the mid-course phase is a topic widely investigated in literature. The developed target identification algorithms are mainly based on the different micro-motions exhibited by ballistic targets. Specifically, the warheads are typically spin-stabilized to ensure that they do not deviate from the intended ballistic trajectories [3]. However, warheads exhibit precession and nutation motion as effect of the Earth gravity. By contrast, decoys wobble when released by the missiles as effect of the atmospheric resistance, the gravity and to the absence of a spinning motor [2]. The micro-motions exhibited by the target lead to range migrations of its principal scattering points observable through a High Resolution Range Profile (HRRP) frame obtained by a wide-band radar. In particular, in [4] an analysis on the capability to discriminate between different target shapes and micro-motions (such as precession, wobbling and tumbling) is conducted by a graphical analysis which combines information extracted from the HRRP frame and a Time-Frequency Distribution (TFD) of radar echoes.

Since the effect of a rotating scatterers in the range-slow time domain is equivalent to the Radon Transform (RT) of target scatterers space distribution function, the Inverse Radon Transform (IRT) is proposed in the literature to reconstruct a 2-D image of the target as a back-projection approach. Specifically, in [5] two IRT based methods are presented for image reconstruction of rotating parts of a target e.g. airplane or helicopter rotor. By contrast, the authors in [6] describe a new approach for cleaning the ISAR image of a target from its rotating parts applying the IRT on a frame of target rangeprofiles. In particular, the rotation period is firstly estimated, then from the IRT of the range profile frame the contribution of rotating parts is detected and filtered out.

In this paper a novel IRT based approach for Automatic Target Recognition (ATR) is presented with the aim to classify targets in a BMD scenario from a sequence of HRRPs. Specifically the IRT of the HRRP frame leads to a 2-D target signature containing information on target motions and the space distribution of its principal scattering points. Then, a feature vector is extracted from the target signature, whose elements are the pseudo-Zernike (pZ) moments extracted from the 2-D target signature. The $\mathrm{pZ}$ moments are very attractive for image classification for their useful properties, such as scale, translation and rotation invariance [7]. For this specific classification approach the rotation invariance is fundamental to ensure robustness with respect to the initial phase of the target micro-motions.

The remainder of the paper is organized as follows. Section II introduces the target model for BM warheads and decoys. Section III describes the proposed classification framework. In Section IV the effectiveness of the proposed algorithm is demonstrated and the performance obtained from simulated data are shown. Section V concludes the paper.

\section{TARGET MODEL}

The BM warheads and decoys exhibit different micromotions during their trajectories which lead to periodic variations of the aspect angle between the radar Line Of Sight (LOS) and the target symmetric axis. Considering the coordinate system $(\hat{X}, \hat{Y}, \hat{Z})$ shown in Figure 1, with origin in the mass centre of the target and such that the position vector of radar lays on plane $\hat{X} \hat{Z}$ and the rotational angular velocity $\boldsymbol{w}_{r}$ direct to $\hat{Z}$-axis, the aspect angle $\alpha=\alpha(t)$ is given by [8]:

$$
\alpha(t)=\cos ^{-1}\left(\sin (\beta) \sin (\theta) \cos \left(\Omega_{r} t+\phi\right)+\cos (\beta) \cos (\theta)\right)
$$


where $\Omega_{r}=\left\|\omega_{r}\right\|$ is the rotation angular velocity, $\theta$ the precession angle, $\beta$ the radar position angle with respect to the $\hat{Z}$-axis and $\phi$ is the initial phase of rotation. Although the precession motion of BM warheads is composed by a spinning motion along the symmetric axis and by conical rotation, only the latter is taken into account in this analysis since for a rotationally symmetric target, the spinning does not change the radar view of the target.

The tumbling of decoys is defined as a rotation of the target such that the symmetric axis is perpendicular to the rotation angular velocity. The aspect angle expression for decoys is obtained by substituting $\theta=90^{\circ}$ in (1). In this work it is assumed that both $\beta$ and $\theta$ are constant during the observation time, therefore the variation of the aspect angle is due to the considered micro motions of the targets.

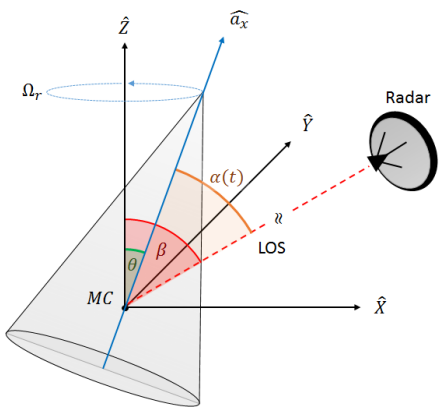

Figure 1: Coordinate system $(\hat{X}, \hat{Y}, \hat{Z})$

In this paper three target shapes are considered: cone, cylinder and cone plus cylinder (see Figure 2). The number of scattering points depends on the target shape. Specifically, for a conical target three principal scattering points are considered: the first is in correspondence of the cone tip; the other two points are located on the intersection between the circumference at cone bottom and the incident plane $\hat{x} \hat{z}$, defined so that it contains both the LOS and the symmetric axis (see in Figure 2a). The cylindrical target is represented by four principal scattering points, specifically two for each base, taken by intersecting the circumferences at the bases and the incident plane (see Figure $2 b)$. Finally, for a target composed by a cone and a cylinder which share the base, five scattering points are considered. One represents the tip of the cone, while the other four are taken on the circumferences in correspondence of the cylinder bases on the incident plane (see Figure 2c).

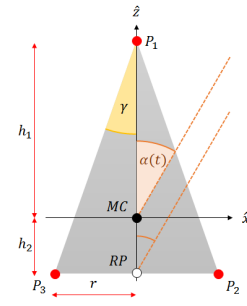

(a)

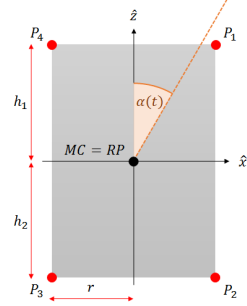

(b)

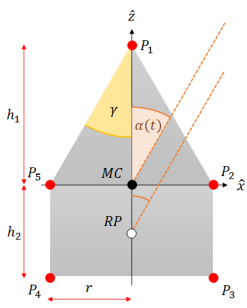

(c)
Figure 2: Target shape model: (a) Cone; (b) Cylinder; (c) Cone plus Cylinder.

For simplicity an approximation for the coefficients of the scattering point is considered in the following analysis, according to which the modulus of coefficients is equal to 1 when there is LOS between radar and the scattering points, while it is 0 when occlusion occurs. The phase of each coefficient depends on the relative distance between the mass centre and the scattering point projected onto the LOS. Specifically, the phase of the $i$-th coefficient can be written as function of the signal carrier frequency, $f$, and of the aspect angle, as follows:

$$
\rho_{i}=\rho_{i}(f, \alpha(t)) \simeq \frac{4 \pi f}{c}\left[x_{i} \sin \alpha(t)+z_{i} \cos \alpha(t)\right]
$$

where $\left(x_{i}, z_{i}\right)$ are the coordinates of the $i$-th scattering points onto plane $\hat{x} \hat{z}$.

\section{Algorithm}

In this section a novel classification algorithm which is able to extract reliable feature based on the micro-motions exhibited by BTs is presented. Specifically the algorithm is based on the IRT and the evaluation of pseudo-Zernike (pZ) moments of a 2-D target signature. Figure 3 represents a scheme block of the presented algorithm.

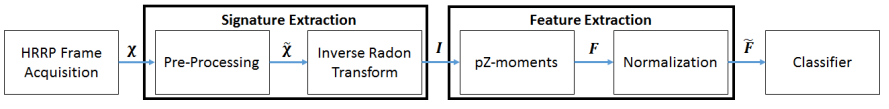

Figure 3: Algorithm block scheme.

The aim of the first block is to generate a HRRP frame by using a Stepped Frequency Waveforms (SFWs) radar data, whose time duration is approximately as long as the period of the rotational motion exhibited by the target. Therefore an accurate estimation of the main rotation rate of target micromotion is needed. In the literature several method for the estimation of the rotation rate $\widehat{\Omega_{r}}$ of a target are presented [9], [10], [11], [12]. However, the rate estimation processing is out of the scope of this work. The number $\widehat{M}$ of bursts needed for computing the target classification depends on the estimated rotation rate value $\widehat{\Omega_{r}}$ and the SFWs radar parameters. Specifically it follows

$$
\widehat{M}=\left\lceil\frac{\widehat{\Omega_{r}}}{2 \pi \mathrm{BRF}}\right\rceil
$$

where BRF is the Burst Repetition Frequency, which is the number of the entire sub-pulses sequences transmitted in a second. It is worth noting that this leads to an approximation error due to the fact that the number of bursts to cover a rotation period is not generally an integer.

The signature extraction block is composed by two steps (see Figure 3). The Pre-processing block consists of two sub-steps. The first is the normalization of each HRRP which makes up the frame, with respect to its own maximum value. The second sub-step consists into re-sizing the normalized frame $\bar{\chi}$ around the range of mass centre, $R_{M C}$, such that the interval of considered ranges is greater than the maximum dimension of targets of interest. Then the target signature $\boldsymbol{I}$ is obtained by normalizing the IRT of $\tilde{\chi}$ with respect to the frame dimension $\hat{M}$. In this work, the filtered back-projection method with ramp filter is considered to realize the IRT. In the hypothesis that the principal motion of the target is compensated, the micromotions exhibited by target leads to a periodic trend in range of the echo from each scattering point in the range-slow time 
domain. Figure 4 shows the range maps of the three scatterers of a cone for a whole rotation period, $T_{r}$, for different couples $(\beta, \theta)$ and their IRT.

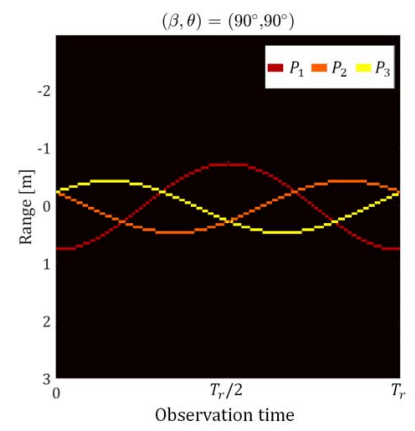

(a)

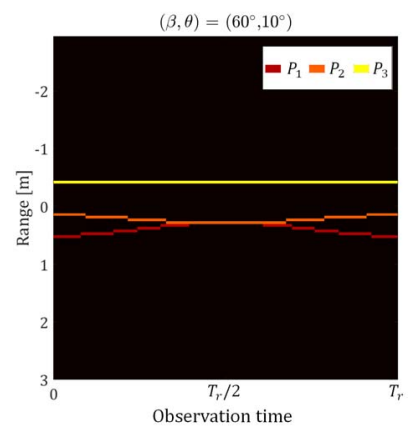

(c)

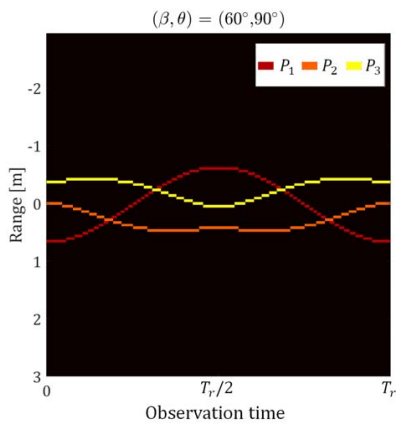

(e)

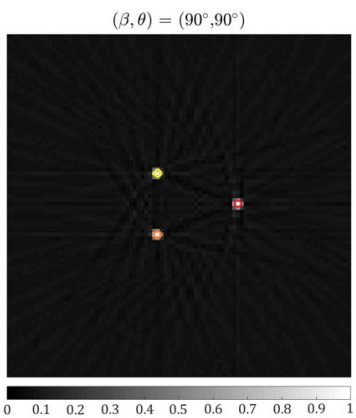

(b)

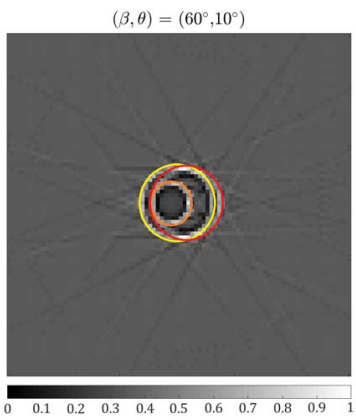

(d)

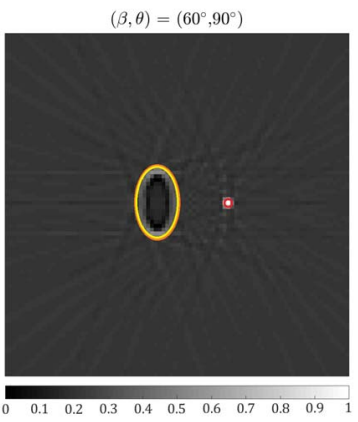

(f)
Figure 4: Range map and its IRT of the three scatterers of a cone for an entire rotation period, $T_{r}$, for different couple of values of $(\beta, \theta)$ : (a) range map and (b) its IRT for $\left(90^{\circ}, 90^{\circ}\right)$; (c) range map and (d) its IRT for $\left(60^{\circ}, 10^{\circ}\right)$; (e) range map and (f) its IRT for $\left(60^{\circ}, 90^{\circ}\right)$.

It is highlighted that each scattering point generates a sinusoidal path centred into $\Delta R$ in the HRRP frame when $\alpha(t)$ varies into $[0, \pi]$, as shown in Figure $4 \mathrm{a}$. Then applying the IRT, all the energy recovered from the path of a single scatterer is concentrated into a point obtaining an image which represents the profile of the object with the exact relative distances between scatterers onto plane $\hat{x} \hat{z}$ (which represents the ISAR image of the object shown in Figure 4b). However from (1) it is clear that $\alpha(t)$ generally varies sinusoidally into $[|\theta-\beta|,|\theta+\beta|]$. Then each scatterer generally moves on different periodic paths. In this specific case, applying the IRT the energy from each path is dispersed into the final 2$\mathrm{D}$ image, such that each of them generates a close line, e.g. circumference or ellipse. For example, Figure $4 d$ shows the IRT of the range map from a precessing cone, in which each scatterer leads to a different circumference, while Figure $4 \mathrm{f}$ shows the IRT of the range map from a tumbling cone, where the contribution from the cone tip is concentrated in a point, while the points on the base generate an ellipse. Therefore, the IRT of HRRP frame can represent the target signature since the close lines are strictly related to the coordinates of scattering points onto plane $\hat{x} \hat{z}$.

Since the HRRP frame of a target can be considered as the RT of the target scattering points distribution function, the initial phase of the micro-motions leads to a rotation of the final target signature in agreement with the RT propriety about linear shifting of the input function [13]. For this reason the choice of reliable features which are invariant for rotation are of interest in this classification problem. Specifically, the pZ moments are geometrical moments with several properties, among which is that their modulus is rotational invariant. Therefore, in this work the $\mathrm{pZ}$ moments are computed in order to extract the feature vector.

Introduced in [14], the pZ moments of order $o$ and repetition $l$ of a 2-D image $\mathcal{I}(x, y)$ are calculated by projecting the image on a basis of 2-D polynomials, $W_{o, l}$, defined on the unit circle, as follows

$$
\zeta_{o, l}=\frac{o+1}{\pi} \int_{0}^{2 \pi} \int_{0}^{1} W_{o, l}^{*}(\rho, \theta) \mathcal{I}(\rho \cos \theta, \rho \sin \theta) \rho \mathrm{d} \rho \mathrm{d} \theta
$$

The presented algorithm computes $(O+1)^{2} \mathrm{pZ}$ moments, where $O$ is the maximum order by projecting the 2-D target signature $\hat{\boldsymbol{I}}$ on the pZ polynomials, and obtaining a feature vector whose $z$-th element is

$$
F_{z}=\left|\zeta_{o, l}\right|
$$

where $o=l=0, \cdots, O-1$ and $z=0, \cdots,(O+1)^{2}-1$. Since the $\mathrm{pZ}$ moments are defined on the unit circle, the signature $\tilde{\boldsymbol{I}}$ is inscribed in the unit circle [7]. Finally, in order to avoid that polarized vector may affect the classification process, the feature vector $\boldsymbol{F}=\left[F_{0} F_{1} \cdots F_{\mathcal{Z}-1}\right]$, with $\mathcal{Z}=(O+1)^{2}$ is normalised as follows:

$$
\tilde{\boldsymbol{F}}=\left(\boldsymbol{F}-\eta_{\boldsymbol{F}}\right) / \varsigma_{\boldsymbol{F}}
$$

where $\eta_{\boldsymbol{F}}$ and $\varsigma_{\boldsymbol{F}}$ are the statistical mean and the standard deviation of the vector $\boldsymbol{F}$, respectively.

The classification performances of the extracted feature vectors are evaluated using a the $k$-Nearest Neighbour $(k$-NN) classifier for its capability to give as output the scores for each class and for its low computational load.

\section{PERFormance AnAlysis}

In this section the performance of the proposed classification algorithm is evaluated with simulated data. The algorithm is tested considering three possible shapes for the BTs which are the cone, the cylinder and the cone plus cylinder. The cone and the cylinder have the same height and radius which are 1 $\mathrm{m}$ and $0.375 \mathrm{~m}$, respectively. The third shape is obtained by joining a cone whose height and radius are $1.4 \mathrm{~m}$ and $0.2 \mathrm{~m}$, respectively, and a cylinder with a height of $0.7 \mathrm{~m}$ and radius $0.2 \mathrm{~m}$. Six classes are considered, each of them corresponding to a particular shape and motion: precessing cone; tumbling 
cone; precessing cylinder; tumbling cylinder; precessing cone plus cylinder; tumbling cone plus cylinder.

Generally the precession angle of warheads with a conical shape is relatively small compared to the half cone angle [3] and its value is generally within $\left[4^{\circ}, 12^{\circ}\right]$ [8]. In the following performance analysis, the precessing classes for each shape is obtained by fixing the precession angle $\theta$ equal to $10^{\circ}$, while for the tumbling classes $\theta=90^{\circ}$.

Both the training and testing sets are simulated considering a SFWs radar transmitting bursts composed by 128 square subpulses with a total bandwidth of $800 \mathrm{MHz}$ between $2.6 \mathrm{GHz}$ and $3.4 \mathrm{GHz}$ with a PRF of $20 \mathrm{kHz}$. The training set for each class is realized for different values of the radar position angle $\beta_{u}=u 5^{\circ}$ with $u=1,2, \cdots, 18$. Each sample of training set is obtained considering the target stopped during the acquisition of a single burst in absence of noise. Specifically for a each $\beta_{u}$ a 360 long HRRP frame is simulated such that the target has completed a rotation of $1^{\circ}$ between two sequential bursts with respect to its motion. Finally the initial phase of rotation is set equal to 0 . The testing set is realized considering noisy observations and continuously moving targets, even during a single burst. In particular, since the warhead spinning and decoy tumbling frequencies are typically smaller than $3 \mathrm{~Hz}$ [15], the dataset for testing each class is realized on varying the rotation rate within $[0.25,3] \mathrm{Hz}$. Then, the angular rotation velocities considered are $\Omega_{r_{v}}=[0.5 \pi+0.25 v \pi] \mathrm{rad} / \mathrm{s}$, with $v=0, \cdots, 22$. From (3) it is highlighted that the observations for different values of the angular rotation velocity are different for the number of bursts composing the HRRP frame. Figure 5 shows how the number of bursts within a rotation period varies with the rotation velocity for the considered SF radar waveform.

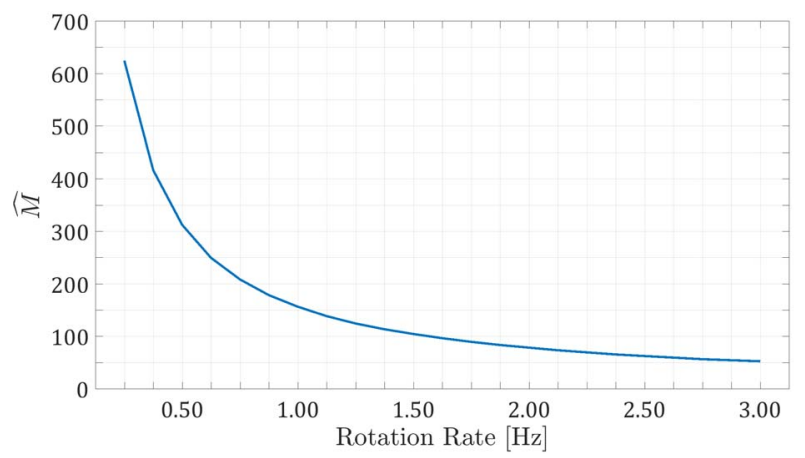

Figure 5: Number of bursts for obtaining the HRRP frame on varying the angular rotation velocity and for the considered SF radar.

The dataset for the test of each class for a fixed noise power and rotation rate is composed by 900 samples. The latter are obtained by realizing 20 acquisitions for each value of $\beta_{\epsilon}=\epsilon 10^{\circ}$ with $\epsilon=1,2, \cdots, 9$, which are different for the noise observation and for the initial phase of the micromotions. The initial phase is drawn randomly from a uniform distribution, within $[0,2 \pi]$.

The performance of the proposed algorithm are evaluated in terms of: Probability of correct Motion identification $\left(P_{M}\right)$, which represents the capability to distinguish between precessing and tumbling targets; Probability of correct Shape identification $\left(P_{S}\right)$, which represents the capability to distinguish between the shapes of targets; Probability of correct
Classification $\left(P_{C}\right)$, the capability to identify the motion and the actual shape of the target.

The analysis is conducted on varying the Signal to Noise power Ratio (SNR), referring to the noise power at the output of the matched filter, and considering the RCS oscillation according to lognormal distribution with zero mean and variance equal to 0.4 [12]. The mean of the three probabilities for each couple of values of SNR and rotation rate is evaluated with a Monte Carlo approach over $10^{4}$ different runs in which 100 samples for each class are randomly taken from the testing dataset and classified. The $k$ value of the $k$-NN classifier is set equal to 1 .

Figure 6 shows the performance obtained on varying the SNR and the angular rotation rate. In order to reduce the distortion in the HRRP due to the variation of the aspect angle within the burst interval a Hamming window is used. It is observed that the performance in terms of the three

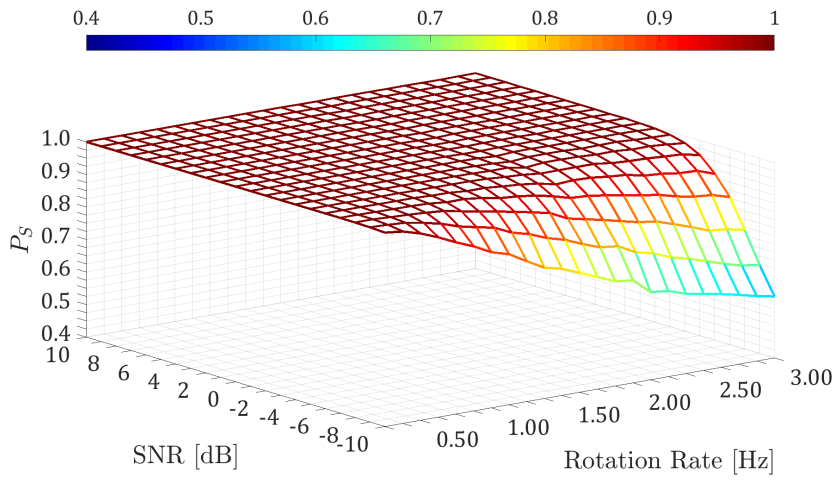

(a)

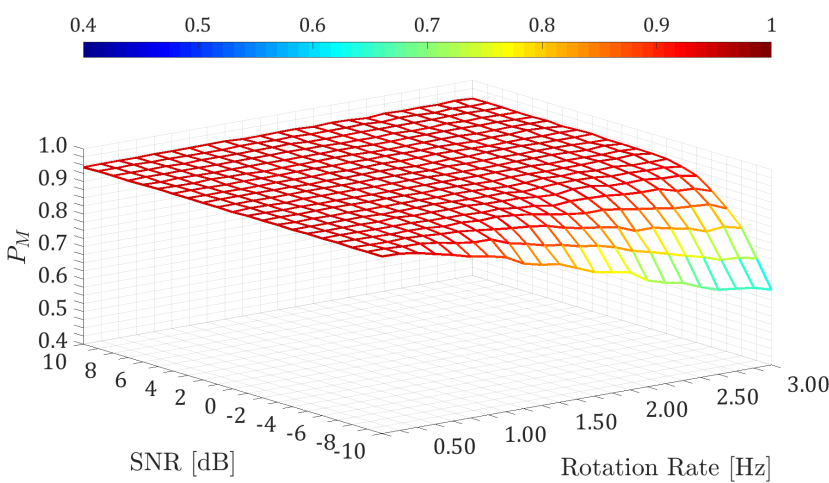

(b)

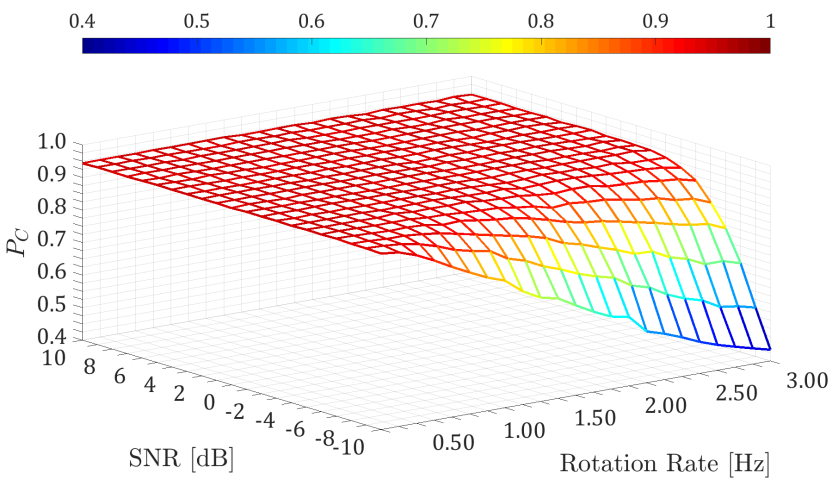

(c)

Figure 6: Performance in terms of $P_{S}$ (a), $P_{M}$ (b) and $P_{C}$ (c). 
probabilities increases as the SNR increases and decreases as the rotation velocity increases. The main reason is that the IRT integrates incoherently the HRRPs which compose the frame, increasing the SNR of the final image. This incoherent processing gain depends on the frame dimension: longer is the HRRP frame, higher is the processing gain. However Figure 6a shows that $P_{S} \geq 0.99$ for SNR greater than $-5 \mathrm{~dB}$ for all the considered rotation rates. $P_{C}$ and $P_{M}$ are very similar for SNR greater than $-5 \mathrm{~dB}$ since $P_{S}$ is close to 1 . Specifically, for these SNR values $P_{C}$ and $P_{M}$ varies within [0.93, 0.95] for all the rotation rates. It is worth noting that the performance in terms of motion recognition and correct classification are affected by the fact that the aspect angle varies in the same way when the values of the angles $\beta$ and $\theta$ are switched. In this analysis there is a case in which precession and tumbling lead to the same variation of aspect angle. Specifically, since the training set for each class is composed by 18 feature vectors, the ambiguity in the motion classification is $1 / 18$, which leads to a maximum value for $P_{M}$ of about 0.95 .

The rotation rates of precession and tumbling are generally different. In fact while the warhead spinning and the decoy tumbling frequency may be similar, the precession frequency is typically an order of magnitude smaller with respect to the spinning [3]. Then the system capability in terms of motion recognition may be improved considering also the estimated rotation velocity. For this reason the capability to recognize the target shape is considered the most relevant in this analysis. In fact the identification of the shape may be discriminant between warheads and decoys allowing also to understand which kind of warheads the target can be (cone plus cylinder can represent a warheads with an additional booster for manoeuvring).

Finally, it is important to point out that the classification algorithm is independent on initial phase of micro-motion and robust with respect to the receiver noise, the RCS scintillation and the approximation error on the HRRP frame dimension.

\section{Conclusion And Future Work}

In this paper a novel framework for the radar classification of BTs has been presented with the aim to distinguish between warheads and decoys. The presented algorithm employs the information relative to the range migrations of the principal target scatterers due to the micro-motions, which are directly observable from a HRRP frame. The presented algorithm is based on the use of RT applied on the HRRP frame received from the target in order to extract a 2-D target signature. A feature vector for the final classification is evaluated by computing the pZ-moments from the 2-D target signature, guaranteeing classification being independent on the initial phase of the target micro-motions (no synchronization required). The effectiveness of proposed approach is tested on simulated SFWs radar data. The dataset for testing the algorithm has been realized for different values for the micro-motion parameters (e.g rotation velocities and precession angle), radar position angle and noise power. The results have shown that the framework allows to discriminate between warheads and decoys with a satisfactory degree of correct classification. The features are robust with respect to the SNR, the RCS oscillation and the HRRP distortions due to micro-movements. Specifically, this algorithm performs well in noise because the IRT has a high accumulation gain to sinusoidal curves in the target signature. The design of a suitable model according to the target of interest (in terms of shape and dimension) and radar system parameters (e.g. polarization and bandwidth) can lead to a model based classification algorithm guaranteeing high performance when preliminary observations of the targets of interest are unavailable.

\section{ACKNOWLEDGMENT}

This work was supported by the Engineering and Physical Sciences Research Council (EPSRC) Grant number EP/K014307/1; and the MOD University Defence Research Collaboration (UDRC) in Signal Processing

\section{REFERENCES}

[1] A. Sessler, J. Cornwall, B. Dietz, S. Fetter, S. Frankel, R. Garwin, K. Gottfried, L. Gronlund, G. Lewis, T. Postol, and D. Wright, "Countermeasure: A technical evaluation of the operational effectiveness of the planned us national missile defense system," Union of Concerned Scientists MIT Security Studies Program, Tech. Rep., April 2000.

[2] A. R. Persico, C. Clemente, D. Gaglione, C. V. Ilioudis, J. Cao, L. Pallotta, A. D. Maio, I. Proudler, and J. J. Soraghan, "On model, algorithms, and experiment for micro-doppler-based recognition of ballistic targets," IEEE Transactions on Aerospace and Electronic Systems, vol. 53, no. 3, pp. 1088-1108, June 2017.

[3] I. Bankman, E. Rogala, and R. Pavek, "Laser radar in ballistic missile defense," Johns Hopkins APL Technical Digest, vol. 22, no. 3, pp. 379393, 2001.

[4] P. Lei, K. 1. Li, and Y. x. Liu, "Feature extraction and target recognition of missile targets based on micro-motion," in Signal Processing (ICSP), 2012 IEEE 11th International Conference on, vol. 3, Oct 2012, pp. 1914-1919.

[5] X. Bai, F. Zhou, M. Xing, and Z. Bao, "High resolution isar imaging of targets with rotating parts," IEEE Transactions on Aerospace and Electronic Systems, vol. 47, no. 4, pp. 2530-2543, OCTOBER 2011.

[6] Y. Hua, J. Guo, and H. Zhao, "The usage of inverse-radon transformation in isar imaging," in 2014 IEEE International Conference on Control Science and Systems Engineering, Dec 2014, pp. 167-170.

[7] C. Clemente, L. Pallotta, I. Proudler, A. De Maio, J. Soraghan, and A. Farina, "Pseudo-zernike-based multi-pass automatic target recognition from multi-channel synthetic aperture radar," Radar, Sonar Navigation, IET, vol. 9, no. 4, pp. 457-466, 2015.

[8] H. Sisan, Z. Jianxiong, Z. Hongzhong, and L. Daobin, "Estimating the precession angle of ballistic targets in midcourse based on hrrp sequence," in 2008 IEEE Radar Conference, May 2008, pp. 1-4.

[9] L. Liu, M. Ghogho, D. McLernon, and W. Hu, "Ballistic missile precessing frequency extraction based on maximum likelihood estimation," in 2010 18th European Signal Processing Conference, Aug 2010, pp. 1562-1566.

[10] X. Bai and Z. Bao, "High-resolution 3d imaging of precession coneshaped targets," IEEE Transactions on Antennas and Propagation, vol. 62, no. 8, pp. 4209-4219, Aug 2014.

[11] H. Yan, X. Fu, X. Lei, P. Li, and M. Gao, "Parametric estimation of micro-doppler on spatial precession cone," in Proceedings of 2011 IEEE CIE International Conference on Radar, vol. 1, Oct 2011, pp. 613-616.

[12] L. Kangle, J. Weidong, L. Yongxiang, and L. Xiang, "Feature extraction of cone with precession based on micro-doppler," in 2009 IET International Radar Conference, April 2009, pp. 1-5.

[13] S. Deans, The Radon Transform and Some of Its Applications, ser. Dover Books on Mathematics Series. Dover Publications, 2007. [Online]. Available: https://books.google.co.uk/books?id=xSCc0KGi0u0C

[14] A. Bhatia and E. Wolf, "On the circle polynomials of Zernike and related orthogonal sets," in Mathematical Proceedings of the Cambridge Philosophical Society, vol. 50, no. 01. Cambridge Univ Press, 1954, pp. 40-48.

[15] L. Li-hua, W. Zhuang, and H. Wei-dong, "Precession period extraction of ballistic missile based on radar measurement," in 2006 CIE International Conference on Radar, Oct 2006, pp. 1-4. 